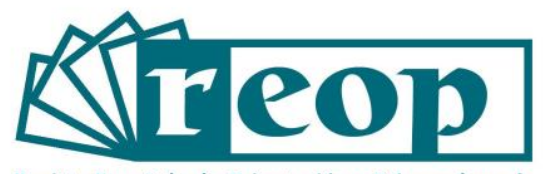

Revista Española de Orientación y Psicopedagogía

\title{
UN ANÁLISIS CUALITATIVO DE LA MOTIVACIÓN ANTE EL APRENDIZAJE DE ESTUDIANTES DE EDUCACIÓN SECUNDARIA
}

\section{A QUALITATIVE ANAL YSIS OF THE MOTIVATION TO LEARN FROM SECONDARY EDUCATION STUDENTS}

\begin{abstract}
Sandra Vázquez-Toledo ${ }^{1}$
Universidad de Zaragoza. Facultad de Educación. Departamento de Ciencias de la Educación. Zaragoza, España

Cecilia Latorre-Cosculluela ${ }^{2}$

Universidad de Zaragoza. Facultad de Ciencias Humanas y de la Educación. Departamento de Ciencias de la Educación. Huesca, España

\section{Marta Liesa-Orús ${ }^{3}$}

Universidad de Zaragoza. Facultad de Ciencias Humanas y de la Educación. Departamento de Ciencias de la Educación. Huesca, España
\end{abstract}

\section{RESUMEN}

Numerosa es la evidencia empírica que ha demostrado que la motivación, además de constituir un factor absolutamente esencial en el proceso de aprendizaje del alumnado, debe preceder a dicho proceso para que, realmente, los estudiantes sientan el deseo real de aprender. Durante la etapa de enseñanza secundaria, la actuación pedagógica y las características personales del propio docente juegan ambas un destacado papel en el proceso motivacional de los jóvenes adolescentes hacia el aprendizaje quienes, en comparación con etapas educativas previas, han ido progresivamente perdiendo los niveles motivacionales que los mantienen voluntariamente próximos a la curiosidad por aprender. En este sentido, se plantea un trabajo de investigación de

\footnotetext{
${ }^{1}$ Correspondencia: Sandra Vázquez-Toledo. Correo-e: svaztol@unizar.es
} 
corte cualitativo con el objetivo de identificar aquellos aspectos didácticos y académicos que inciden en el estado motivacional del alumnado de la etapa de Secundaria y Bachillerato. Para ello, y teniendo en cuenta que el número de jóvenes estudiantes participantes en el estudio es de 28 (comprendidos entre las edades de 12 y 18 años), se conformaron un total de cuatro grupos de discusión con alumnado de tres centros de Educación Secundaria públicos. Los resultados, analizados atendiendo a las categorías resultantes del estudio cualitativo de la información, reflejan un conjunto de cualidades y aspectos a tener en consideración en orientación educativa y, consecuentemente, sobre los que reflexionar con detenimiento. Todo ello, con la firme intención de introducir las oportunas mejoras que conduzcan al alumnado a manifestar un auténtico e intrínseco interés por aprender y formarse a lo largo de la vida.

Palabras clave: Motivación; enseñanza secundaria; orientación educativa; pedagogía; docente.

\section{ABSTRACT}

There is ample empirical evidence showing that motivation, in addition to being an essential factor in the learning process of students, must precede this process so that students feel the real desire to learn. During the stage of Compulsory Secondary Education, the pedagogical action, and the personal characteristics of the teacher both play an important function in the motivational process of young adolescents towards learning. In comparison with previous educational stages, students have progressively lost their motivational levels that keep them voluntarily close to the curiosity to learn. In this sense, a qualitative research is proposed with the aim of identifying those didactic and academic aspects that affect the motivational status of students in Secondary Education. Considering that the number of young students participating in the study is 28 (between the ages of 12 and 18), a total of four discussion groups were formed with students from three public secondary education centres. The results, analyzed according to the categories of the qualitative study of the information, reflect a set of qualities and aspects to be taken into consideration in educational guidance and, consequently, about which to reflect carefully. All this, with the firm intention of introducing the necessary improvements that lead students to manifest an authentic and intrinsic interest in learning and training throughout life.

Key Words: Motivation; Secondary Education; educational guidance; pedagogy; teacher.

\section{Cómo citar este artículo:}

Vázquez-Toledo, S., Latorre-Cosculluela, C. y Liesa-Orús, M. (2021). Un análisis cualitativo de la motivación ante el aprendizaje de estudiantes de educación secundaria. Revista Española de $\begin{array}{llll}\text { Orientación } \quad y & \text { Psicopedagogía, } & 32(1), & 116-131 .\end{array}$ https://doi.org/10.5944/reop.vol.32.num.1.2021.30743

\section{Introducción}

Durante el periodo de la adolescencia, los jóvenes se ven expuestos a numerosos cambios asociados con la etapa de la pubertad, con la transición de la escuela primaria a secundaria y con 
su funcionamiento social, familiar y escolar. Esta transición es considerada un evento significativamente particular dentro del curso de escolarización del alumnado (Hanewald, 2013). De acuerdo con autores como Dotterer y Lowe (2011), factores relacionados con los cambios en el entorno escolar y en la organización, y aquellos relativos a la modificación de los criterios de evaluación, confluyen todos ellos y obligan a los jóvenes a iniciar una reevaluación de sus propios sistemas de aprendizaje. Para muchos niños que dejan atrás la etapa de Educación Primaria, la naturaleza de los cambios que experimentan puede repercutir, incluso de forma negativa, en su progreso académico durante la adolescencia. En el trabajo de Monarca et al. (2012) se concluye que, al menos para el $33 \%$ de los estudiantes que inician la escolaridad en Secundaria, la transición les supone un proceso de ajuste del que se derivan consecuencias negativas para sus aprendizajes académicos.

Las expectativas, actitudes, creencias y motivaciones del alumnado durante ese periodo de transición se configuran como determinantes de las conductas, perspectivas y comportamientos que manifiestan hacia la etapa de Secundaria que van a iniciar (Engels et al., 2019). Por ello, el éxito de esta transición y de los procesos de adaptación y aprendizaje de los estudiantes va a depender, en gran medida, de la toma en consideración de estos factores por parte de los responsables educativos. Concretamente, y dado que es objeto de análisis del presente estudio, la motivación merece ser considerada con especial atención desde diversas ramas de conocimiento y ámbitos de investigación (Guay et al., 2019).

\section{Antecedentes teóricos}

La motivación constituye, sin duda alguna, un factor claramente decisivo del proceso de aprendizaje y rendimiento de un estudiante (Mega et al., 2014). A edades muy tempranas, los niños manifiestan gran interés por el aprendizaje, pues se encuentran en una etapa de continuos descubrimientos. Sin embargo, y coincidiendo con periodos educativos posteriores, los jóvenes adolescentes se ven implicados en un proceso de pérdida de la motivación que tiene sus raíces en una serie de condiciones (Leong et al., 2018): la complejidad de las materias, los cambios en su entorno y aquellos propios del periodo evolutivo y el funcionamiento escolar, entre algunos otros.

Gran parte de la varianza que se da respecto al rendimiento escolar del alumnado y que no puede ser explicada por otros factores tales como la capacidad intelectual de cada uno, se relaciona con este constructo de motivación, ejemplo de lo cual puede apreciarse en el estudio de Roux y Anzures (2015). En comparación con la inteligencia y otras habilidades más específicas, la motivación puede ser fácilmente influenciable por factores circunstanciales. Por tanto, cuando los profesores tienen como objetivo mejorar el rendimiento de sus estudiantes, potenciar su motivación puede resultar tan primordial como la transmisión de información y conocimientos (Džinović et al., 2019).

La importancia de la motivación en el aprendizaje de adolescentes viene reflejada, por ejemplo, en estudios como el de Caso-Niebla y Hernández-Guzmán (2007) en el que, en base a una muestra de 1582 jóvenes, se encuentran una serie de factores de naturaleza afectivomotivacional que explican, al menos en parte, su rendimiento escolar. En esta línea, se subraya la necesidad de disponer de nuevas estrategias que permitan fomentar la motivación del alumnado hacia el aprendizaje. Estudios como el realizado por Smit, de Brabander et al. (2017) reconocen esta necesidad especialmente en las etapas de Secundaria pues, a medida que progresan a lo largo de los diferentes grados escolares, se ha constatado una clara tendencia hacia motivaciones 
de carácter más extrínseco, decreciendo paulatinamente la motivación intrínseca que, en general, tiene el alumnado durante la etapa de escolaridad Primaria (Froiland y Oros, 2014; Graham et al., 2016).

El creciente aumento del número de jóvenes adolescentes que se ven abocados al fracaso escolar y abandono precoz de los estudios ha traído como consecuencia la aparición de un grave problema al que debe hacer frente el sistema educativo español (Ministerio de Educación y Ciencia, 2007). Esta cuestión, altamente preocupante, ha conducido a diversos responsables educativos e investigadores a reconsiderar seriamente los factores implicados en la falta de interés de este alumnado. De cualquier modo, la motivación académica de los estudiantes es un amplio constructo en el que confluyen multitud de factores y, como tal, su abordaje educativo es complejo (Renaud et al., 2015).

En los últimos años, se han propuesto tres aspectos fundamentales que tienen una significativa influencia en la motivación e implicación de los estudiantes en su proceso educativo (Dogan, 2015; Reeve y Lee, 2014). En primer lugar, la participación en clase, fenómeno esencial en tanto que los entornos de aprendizaje que fomentan la implicación activa de su alumnado mejoran significativamente el compromiso de estos hacia el aprendizaje. El disfrute en la escuela constituye el segundo proceso que repercute en la disposición que presenta el alumnado para asistir a clase y en las metas que tienen respecto a su experiencia académica (Huhtiniemi et al., 2019). Finalmente, y en términos de aspiraciones educativas, aquellos estudiantes con mayores motivaciones y compromisos son más propensos a la implicación en futuros cursos y estudios superiores.

Los factores implicados en la motivación del alumnado no surgen únicamente del propio individuo o exclusivamente del contexto, sino que, por el contrario, se produce una interacción entre las características de cada persona y el ambiente escolar (Pouratashi y Zhu, 2018; Urdan y Schoenfelder, 2006). Por ejemplo, las relaciones e interacciones sociales que se establecen entre los profesores y el alumnado tienen una clara influencia sobre el clima de las clases. Los docentes son, según Urdan y Schoenfelder (2006), los responsables de regular el funcionamiento académico, los enfoques de aprendizaje y el modo en que los chicos se comunican. Por ello, las percepciones de los estudiantes sobre el estilo de enseñanza de sus profesores, la metodología que emplean y el ambiente de las aulas, repercuten en el modo en que los jóvenes aprenden y en sus actitudes hacia los retos académicos que se les propone resolver.

La relevancia de la actuación del profesor está probada en trabajos de investigación como el de Turner et al. (2002), en el que se analizan las variables de instrucción relacionadas con las conductas evitativas de los estudiantes hacia la asignatura de matemáticas. Del análisis cualitativo que llevan a cabo los autores se desprende una idea fundamental, y es que aquellas aulas en las que imperan conductas de poca evitación entre su alumnado presentan, como característica principal, un ambiente de enseñanza en el que se les permite demostrar sus habilidades, se les motiva con diferentes refuerzos y se les ofrece ayuda para la comprensión de aspectos más complejos. Comportamientos docentes y de la figura de los líderes tales como la asertividad y la capacidad de respuesta son considerados también altamente positivos, por lo que cabe esperar que el alumnado tenga actitudes más positivas hacia sus aprendizajes, trabaje con esfuerzo para aprender y aprecie el contenido de lo que está trabajando (Cudney y Ezzell, 2018). Los resultados de trabajos como el de Kunter et al. (2013) constatan que, cuando los profesores insisten en valorar positivamente los aprendizajes, la comprensión de los contenidos y la persecución de tareas desafiantes del alumnado, estos perciben unas metas a lograr mucho más consolidadas y, por ende, un grado más elevado de motivación para aprender.

Bajo los hallazgos hasta el momento expuestos, y considerando la esencial necesidad de desarrollar continuas y periódicas investigaciones en el ámbito educativo debido a las sucesivas transformaciones que en él se producen, se va a ahondar empíricamente en las perspectivas que estos estudiantes reportan cuando se alude a una de las figuras principales que condicionan su 
motivación ante el aprendizaje: el docente y su función. Parece interesante, por tanto, plantearse preguntas de investigación tan relevantes como las siguientes: ¿qué aspectos influyen en la pérdida de motivación de los estudiantes en etapas educativas correspondientes a Secundaria y Bachillerato? ¿Cuáles son las percepciones específicas que, según el alumnado, explican su falta de motivación ante el aprendizaje? El propósito central del presente estudio es, por tanto, dar respuesta a un proceso investigativo que ofrezca una clara visión sobre las percepciones de las que informan unos de los principales agentes de los sistemas escolares acerca de los elementos que, en la actualidad, están repercutiendo en la pérdida de los niveles de motivación que se estimarían oportunos para iniciar, adquirir e interiorizar adecuadamente el aprendizaje. En términos más específicos, se pretende identificar los aspectos didácticos y académicos que inciden en el descenso de la motivación del alumnado de Secundaria y Bachillerato, con las siguientes actuaciones: a) conocer qué factores están involucrados en la motivación hacia el aprendizaje desde la perspectiva del alumnado; b) indagar en el análisis del modo en que la actuación pedagógica y las características del docente condicionan la motivación; c) comprender las necesidades de los estudiantes hacia el aprendizaje; y d) reflexionar sobre los cambios necesarios para revertir la desmotivación del alumnado en las etapas de Secundaria y Bachillerato.

\section{Método}

La metodología que fundamenta este estudio tiene una orientación eminentemente cualitativa, en tanto que se preocupa por la construcción del conocimiento sobre y a partir de la realidad sociocultural desde el punto de vista de los actores sociales (Harreveld et al., 2016; SánchezGómez et al., 2019). En el marco educativo, y de acuerdo con Cerrón Rojas (2019), este aspecto se traduce en comprender los fenómenos a través de las interpretaciones y percepciones de los sujetos que intervienen en la acción educativa. Se estudia la realidad sin fragmentarla y contextualizándola, obteniendo conclusiones generales a partir de situaciones concretas.

\section{Muestra}

La selección de los participantes fue no probabilística e intencional, respondiendo así a una serie de criterios previamente definidos. De este modo, en el estudio han participado un total de 28 estudiantes comprendidos entre las edades de 12 y 18 años, y pertenecientes a tres institutos públicos de una provincia de la zona norte de España. Se consideraron elegibles todos aquellos estudiantes que estuvieran matriculados en cualquier curso contenido entre primero de la E.S.O. y segundo de Bachillerato. Una vez se hubo anunciado en estos tres institutos el tipo de investigación que iba a realizarse, se permitió que fuera la voluntariedad de participación en el estudio la que conformase inicialmente la muestra.

De la población diana inicial, la selección de esta muestra definitiva se fundamentó en las orientaciones de Canales y Peinado (1994), quienes recomiendan que sea heterogénea, en tanto que un grupo con sujetos muy similares podría producir un discurso muy redundante en los grupos de discusión, técnica de producción de datos adoptada en este trabajo. Con la pretensión de atender a este requerimiento, se ha incluido alumnado de diferentes cursos de las etapas de Secundaria y Bachillerato distribuidos del siguiente modo: 7 estudiantes de primer curso de 
Secundaria, 6 estudiantes de tercer curso de Secundaria, 7 estudiantes de primer curso de Bachillerato y, finalmente, 8 estudiantes de segundo curso de Bachillerato. Si bien no se fijó un número exacto de participantes por cada centro de la E.S.O. y Bachillerato, también se intentó asegurar cierto equilibrio en él. Los tres centros tenían características similares en lo que respecta al alumnado que a él acuden (clase socioeconómica media) pero se encontraban ubicados en lugares muy dispares de la localidad (en el centro, en las afueras y en un lugar intermedio).

\section{Instrumento}

Se utilizó la estrategia de grupos de discusión, técnica de producción de datos en investigación de corte cualitativo que recurre a la entrevista realizada a todo un grupo de personas para recopilar información relevante sobre el problema del estudio (Bisquerra, 2004, p. 43). Como estrategia de comunicación, los grupos focales resultan de fundamental relevancia porque posibilitan el acceso, de un modo abierto y profundo, a las valoraciones personales que emiten los individuos respecto a las temáticas que se les ofrece cuestionar, abriéndose la ocasión de intercambiar opiniones grupalmente y discusiones que atañen a actitudes, creencias y experiencias (Carey et al., 2012). Fueron, así, un total de cuatro los grupos de discusión conformados.

Como criterios a la hora de generar los grupos de discusión, Guest et al. (2017) defienden que estos combinen unos mínimos de heterogeneidad y homogeneidad. Para el caso que aquí atañe, la homogeneidad vino determinada por el tema objeto de estudio (la motivación hacia el aprendizaje), pues todos los implicados están vinculados a ello por ser estudiantes, por los grupos de edad y por el centro de Secundaria en el que se encontraban matriculados. Se optó por disponer grupos de estudiantes de la misma edad en cada uno de los grupos con la finalidad de analizar, con el mayor detalle y profundidad posible, las implicaciones del discurso de este alumnado en cuanto a la motivación ante el aprendizaje en cada uno de los cursos académicos. A su vez, son grupos heterogéneos en tanto que estos participantes son de distinto género y presentan expedientes académicos variados (información que, previamente al desarrollo de los grupos de discusión, se solicitó a los participantes). En definitiva, se establecieron estos criterios con la esencial finalidad de obtener un discurso enriquecido, quedando los grupos de discusión conformados del modo que aparece en la tabla 1 :

\section{Tabla 1}

Características de los grupos de discusión.

\begin{tabular}{cclc}
\hline $\begin{array}{c}\text { Grupo } \\
\text { focal }\end{array}$ & Curso & \multicolumn{1}{c}{ Participantes } & \multicolumn{1}{c}{$\begin{array}{c}\text { Edad de los } \\
\text { participantes }\end{array}$} \\
\hline 1 & $1^{\circ}$ E.S.O. & $\begin{array}{l}4 \text { alumnas } \\
3 \text { alumnos (Uno de ellos repetía curso) }\end{array}$ & Entre 12 y 13 años \\
\hline 2 & $3^{\circ}$ E.S.O. & $\begin{array}{l}3 \text { alumnas } \\
3 \text { alumnos }\end{array}$ & Entre 15 y 16 años \\
\hline 3 & $1^{\circ}$ Bachillerato & $\begin{array}{l}4 \text { alumnas } \\
3 \text { alumnos }\end{array}$ & Entre 16 y 17 años \\
\hline 4 & $2^{\circ}$ Bachillerato & $\begin{array}{l}4 \text { alumnas } \\
4 \text { alumnos (uno de ellos repetía curso de forma } \\
\text { voluntaria para subir nota) }\end{array}$ & Entre 17 y 18 años \\
\hline Total & & 28 &
\end{tabular}

\footnotetext{
Fuente: Elaboración propia.
} 


\section{Procedimiento}

Por cuestiones de disponibilidad espacial y temporal, se optó por realizar los grupos de discusión sucesivamente, fuera del horario escolar habitual, pero en aulas ubicadas dentro de los mismos institutos. En aras de facilitar el trabajo de los grupos, se ideó un guion semi-estructurado que aparece en la tabla 2, y el desarrollo de cada uno de ellos estuvo orientado por una moderadora (investigadora 1) que condujo el curso de las emisiones comunicativas de los participantes. El guion fue previamente consensuado por las tres investigadoras a partir del cuerpo de literatura revisado acerca de la temática objeto de estudio. El papel fundamental de esta moderadora era posibilitar la exposición de ideas y vivencias de los participantes respecto al asunto planteado con total libertad.

Para asegurar la fiabilidad en la conducción del debate desarrollado, los cuatro grupos de discusión estuvieron dirigidos por la misma moderadora. Tras la presentación del objetivo general del estudio, se definieron los conceptos preliminares del tema a investigar. En dicho proceso aclaratorio inicial, se construyó una definición junto con los participantes del concepto de motivación ante el aprendizaje, lo que sirvió además para crear un clima de confianza que permitiera un acercamiento entre la moderadora y los estudiantes. El debate fue iniciado a través del lanzamiento de una pregunta exploratoria general: ¿Cómo valoráis vuestra actual motivación ante el aprendizaje? En la tabla 2 puede consultarse el guion temático aproximativo seguido en los grupos de discusión. Finalizado el periodo de discusión, se solicitó a cada estudiante participante un resumen de aquellos aspectos de mayor interés y relevancia. La moderadora de los grupos fue anotando, además de las observaciones de los participantes, sus propias impresiones respecto al curso de desarrollo de cada uno de los debates. Siguiendo un criterio de saturación para garantizar la fiabilidad de la información recolectada, se mantuvo el proceso de debate hasta que se consideró que las aportaciones ya habían dejado de añadir nueva información a la discusión.

\section{Tabla 2}

Guion temático inicial de los grupos de discusión.

\section{Escenario de discusión}

\section{Preguntas exploratorias generales}

¿Cómo valoráis vuestra actual motivación hacia el aprendizaje?

¿Qué opináis acerca de la metodología de enseñanza que tienen vuestros profesores (es decir, acerca de "cómo" enseñan)?
Tipo de motivación.

Causas principales del estado motivacional actual ante el aprendizaje.

Motivos por los que se estudia y se acude al instituto.

Características del tipo de enseñanza (aspectos positivos y negativos).

Principales modos en que el profesor presenta los aprendizajes.

Grado en que esta actuación del profesor despierta el interés de los estudiantes.

Acerca de vuestros profesores, de sus Grado de implicación de los profesores en el aprendizaje de características personales especialmente, ¿cómo los consideraríais? los estudiantes.

Estilo de enseñanza adoptado.

Competencias sociales y emocionales.

Priorización de esfuerzos o capacidades en el estudiante.

¿Cómo creéis que, con la actuación Puntos de apoyo para lograr mejoras en el nivel de docente, se podría despertar vuestro interés motivación para aprender.

por lo que aprendéis?

Factores clave a los que hay que prestar especial atención.

Fuente: Elaboración propia. 
Todas las sesiones fueron grabadas, previa autorización de los participantes, con una cámara de vídeo para facilitar la exactitud y precisión en la posterior transcripción de información. Por otra parte, la acción de la moderadora estuvo orientada a garantizar que los participantes intervenían de forma equilibrada durante el desarrollo de los grupos de discusión. Para ello, se sirvió de estrategias de motivación y propias de la moderación de los discursos orales (Del Rio-Roberts, 2011). Salvo que se originasen comentarios despectivos entre los participantes (que no se produjo en ningún momento), la moderadora no emitió juicios de valor, no introdujo opiniones propias (Grønkjær et al., 2011). Si bien dependió de diversas variables (tales como la disposición de los participantes), la duración aproximada de cada grupo de discusión varió entre 45-60 minutos.

Una vez los grupos de discusión se hubieron diseñado, puesto en marcha e implementado, en una primera fase del análisis de información se llevó a cabo un estudio preliminar exploratorio de los textos obtenidos en estos grupos, teniendo en consideración que primeramente estos fueron sometidos a transcripción. Posteriormente, se trataron los discursos colectivos y se sometieron a análisis de contenido del discurso. Tras ello, se agrupó la información en categorías para proceder a vincularla con el cuerpo de la literatura que respalda el trabajo de investigación y poder, de este modo, conformar las categorías emergentes y articular las oportunas conexiones entre dichas temáticas objeto de análisis.

La reducción de las categorías se realizó mediante el principio de saturación hasta que dejaron de emerger nuevas ideas y conceptos sobre la temática objeto de análisis. Consecutivamente, se especificaron las relaciones entre ellos con la finalidad de reducir la cantidad de información recopilada (Vaugh y Turner, 2016).

Finalmente, el último análisis se llevó a cabo mediante el consenso de las representaciones e interpretaciones de las tres investigadoras, siendo una de ellas la moderadora de los grupos de discusión.

\section{Resultados}

Los resultados reflejan una síntesis de las opiniones manifestadas por los participantes de todos los grupos de discusión y las consecuentes interpretaciones de la moderadora y la otra investigadora. En términos generales, la información analizada se presenta estructurada en torno a dos ejes clave: por un lado, aquellos elementos relacionados con la actuación pedagógica que desarrolla el profesor y, por otro lado, las características o competencias propias y personales de la figura del docente.

En un primer momento, el análisis del estado motivacional actual reportado por el conjunto de jóvenes participantes en el estudio revela un sentimiento de queja común acompañado de la manifestación de poco interés hacia su propio aprendizaje, especialmente en aquellas materias que no despiertan en absoluto su agrado. A todo ello, se le añade la escasa participación en el desarrollo de las clases que perciben, lo que les conduce a presentar, en general, una escasa motivación por la formación académica que reciben en los institutos de Educación Secundaria Obligatoria. De hecho, algunos de ellos afirman incluso que la mayoría de las acciones que deciden implementar en su proceso académico, tienen como finalidad última la mera promoción de etapa educativa para evitar la repetición de curso, aspecto que se ve claramente reflejado en la siguiente afirmación que hacía uno de los participantes: 
GR2M4: La verdad es que, muchas veces, estudiamos sólo para pasar de curso, para aprobar los exámenes finales. A lo mejor suena mal, pero es la verdad. Me imagino repitiendo curso y... me asusta bastante.

La percepción de obligatoriedad del proceso educativo les resulta, también, un aspecto molestamente negativo que interfiere en el interés de estos jóvenes por aprender. Por fortuna, y pese a que puede resultar alarmante la cantidad de alumnado que, firmemente, ofrecen todos estos argumentos, se aprecia un elemento positivo en la reflexión que estos hacen sobre la importancia y necesidad del ámbito motivacional en el proceso de aprendizaje y formación:

GR4F: Es importante estar a gusto con lo que estudias, en lo que trabajas y en lo que haces.

Retomando las preguntas concretas de investigación y los objetivos propuestos para el presente estudio, son diversos los elementos a los que los participantes hacen alusión cuando se les pregunta por los factores o aspectos, relacionados con la actuación pedagógica que lleva a cabo el docente, que consideran están afectando negativamente a la motivación que manifiestan hacia sus aprendizajes. Algunos de los participantes apuntan, en primer lugar, a la excesiva valoración que se hace de sus resultados, en lugar de situar el foco de atención sobre el proceso de aprendizaje en sí mismo que, verdaderamente, es en el que se obtienen y asimilan adecuadamente los conocimientos y habilidades trabajadas. Ejemplo de ello lo encontramos en la rotunda afirmación que dos de las estudiantes del nivel de Bachillerato lanzaban:

GR3F: Aquí sólo nos enseñan a aprobar exámenes más que a aprender.

GR3F: De hecho, algunos profesores creen que estamos en clase para aprobar exámenes y no para aprender, lo que implica que estudiar sólo valga para realizar el correspondiente examen.

Directamente vinculado con este aspecto, otra de las temáticas más ampliamente nombrada es aquella relacionada con la excesiva exigencia de memorización de aprendizajes, priorizando este hecho antes que su comprensión, íntegra asimilación y vinculación con otras áreas. Perciben, además, que cada curso académico se trabajan prácticamente los mismos contenidos de aprendizaje que en años previos, atribuyéndoles por tanto a estos una notable monotonía. En consecuencia, la utilidad del contenido de los aprendizajes presentados en el aula se advierte como escasa, pues desconocen para qué finalidad concreta y en qué contextos oportunos van a poder poner en verdadera práctica todo eso supuestamente aprendido memorísticamente. Este hecho podría mantener un estrecho vínculo con el enfoque orientado hacia la preparación de la EBAU (Evaluación del Bachillerato para el Acceso a la Universidad) hacia el que, en la actualidad, se focalizan las dinámicas de aprendizaje en la etapa de Bachillerato. Véase, como ejemplo, el siguiente fragmento de discurso de una de las estudiantes de segundo curso de Bachillerato:

GR4F: Todos los años se nos dan los mismos contenidos. Las clases siempre son iguales. Además, pero ¿para qué me va a servir a mi aprender y memorizar toda esta cantidad de información? Por ejemplo, en la asignatura de Lengua Castellana mismo, ¿de qué nos sirve analizar sintácticamente una frase, si no tengo ni idea de dónde aplicarla después, y para qué me va a servir en la vida exactamente? Terminamos estudiándolo porque "hay que" saberlo, pero no porque "queramos" aprenderlo.

\footnotetext{
${ }^{4}$ Los fragmentos de información correspondiente a las opiniones recogidas durante el desarrollo de los grupos de discusión, se exponen con este formato de código cuyas letras y números tienen el siguiente significado: GR: Grupo; Número (1/2/3/4): Número de grupo focal al que pertenece el participante que ha hecho la emisión verbal; Letras (M/F): Género del participante (M: masculino; F: femenino).
} 
En definitiva, estos jóvenes consideran que la memorización de aprendizajes, así como la realización de exámenes (especialmente aquellos no planificados, con un componente de "sorpresa") a las que se les expone, pueden traducirse en una forma de "castigo" que, en absoluto, estiman como un hecho constructivo que les pueda resultar de utilidad para su formación académica. A este respecto, referido a la temática de los castigos, muchos de estos adolescentes coinciden en tachar de abusivo el número de amonestaciones y reprimendas escritas que, con habitualidad, les son enviadas a sus hogares. La consecuencia que de ellas puede derivarse, además, no la interpretan como un hecho que, ciertamente, les pueda servir para aprender de la situación. Es, para ellos, un mero castigo más con muy poco sentido funcional.

Uno de los ejes centrales que ha sido foco principal del debate originado en los grupos de discusión y que, lógicamente, se incluye en este ámbito de actuación pedagógica, es el referido a la metodología de enseñanza-aprendizaje implementada en las aulas a las que estos jóvenes acuden. Así pues, una gran mayoría de los participantes no ha dudado en apuntar que, entre los factores más importantes que constituyen barreras a la motivación que presentan ante los aprendizajes se encuentran, precisamente, las estrategias y técnicas de enseñanza que el docente adopta en el curso de sus clases. El alumnado pasa a ser partícipe de un proceso tradicional de trasmisión de conocimientos unidireccional y, por tanto, un mero receptor de información, percibiéndolo en argumentos tales como:

GR2M: Creo que las clases duran demasiado tiempo, nos resultan pesadas y, a veces, se hace muy duro aguantar hasta el final manteniendo la atención. Son muchas horas al día sólo escuchando y apuntando cosas en un cuaderno.

GR4F: Explicar solo, y ya está, no es suficiente. Si participáramos más en clase, seguro que las horas en el instituto nos pasarían más rápido.

Por otro lado, y en lo que respecta al desempeño y competencias propias y personales de la figura del docente, los jóvenes destacan, como primera característica de absoluta relevancia, una falta de formación en el área socioemocional de sus profesores, especialmente en el ámbito que concierne a la comprensión y empatía con la figura del estudiante. Directamente vinculado con este aspecto, también aquejan un excesivo autoritarismo por parte de sus profesores que, en ningún caso, perciben como positivo o como un hecho que realmente les conduzca a despertar su motivación en las aulas. La destacada importancia que le conceden a ambos aspectos se ve apoyada, incluso, por la hipótesis de que la motivación proviene más del modo en que el docente presenta el temario a aprender, que de la temática específica que se aborda en cada materia:

GR3F: Yo creo que, lo mejor que puede tener un profesor, es que conozca a todos sus alumnos y que entienda que no todos son iguales.

GR4F: Está bien que tengan autoridad, porque si no... Pero estaría mejor que fueran más cercanos, con menos autoridad, que se rían de vez en cuando con nosotros y que nos feliciten cuando nos lo hemos ganado.

Estos estudiantes de la etapa de Secundaria están dispuestos a comprometerse de forma más activa en su propio proceso de aprendizaje, siempre y cuando perciban por parte de sus profesores de referencia un cercano afecto hacia ellos y una implicación activa en el deseo porque su alumnado aprenda y disfrute en las aulas. Para ellos, también el estado de ánimo de los docentes resulta de importancia en su proceso motivacional, lo que puede apreciarse en el siguiente texto extraído de la información recabada en los grupos de discusión. Perciben, sin lugar a duda, la desmotivación que algunos de los propios profesores manifiestan en las clases.

GR4M: Algunos profesores no controlan la materia que nos dan. Además, a algunos de ellos también se les ve un poco desanimados en el momento de dar las clases, y nosotros nos damos cuenta. 
Además de ello, manifiestan cierta disconformidad respecto al hecho de que raramente se incentiva y apoya realmente a aquel alumnado que se encuentra repitiendo curso. Se advierte la sensación de que son estudiantes que, como han "fracasado" con esa repetición de curso, los esfuerzos invertidos en ofrecerles los soportes que verdaderamente necesitan para continuar adelante resultan en vano. A este respecto, probablemente no resultaría un error ligar esta cuestión con el fenómeno descrito en líneas anteriores cuando se mencionaba la falta de receptividad emocional y, en general, de formación en competencias socioemocionales que los jóvenes atribuyen a sus profesores.

$Y$, ¿cuál es realmente el ideal de profesor que estos adolescentes desearían tener en el aula, y que podría contribuir a que su motivación ante los aprendizajes fuera visiblemente más alta? En la línea de lo señalado por gran parte de los participantes, demandan a un tipo de docente parecido a un "hermano mayor", que sea un guía antes que instructor, y entre cuyos valores éticos y morales prime la cercanía hacia su alumnado en combinación, eso sí, con la serenidad y el saber estar (es decir, con la parte positiva de lo que correspondería al exagerado autoritarismo). Que sea capaz, en definitiva, de conquistar el respeto del conjunto de su alumnado prestando una cuidadosa atención a las necesidades y características que, primeramente, como personas, cada uno de ellos presenta. En un tramo del tiempo final de uno de los grupos de discusión, una de las participantes incluso llega a emitir un mensaje referido a los futuros docentes que, en la actualidad, están completando su formación para ejercer esta profesión:

GR4F: A los futuros profesores yo les diría que se cuiden, que no se rindan, que se adapten un poco a sus alumnos y que no amonesten ni pongan tantas faltas por escrito que no sirven para nada muchas veces.

\section{Conclusiones y Discusión}

A partir del análisis de la información recabada en este estudio y de aquello que muchos otros trabajos de investigación han defendido (Chien y Hsieh, 2018; Tasgin y Coskun, 2018), se ha podido constatar que la motivación que presentan estos jóvenes escolarizados en las etapas educativas de Educación Secundaria Obligatoria y Bachillerato, depende más de la metodología de enseñanza-aprendizaje y la actuación pedagógica adoptada por el docente -lo que bien se conoce como el "cómo se enseña"-, que del contenido en sí mismo que en cada materia se aborda -lo que se conoce como el "qué se enseña"-.

En términos generales, se observa una escasa motivación ante el aprendizaje que, en muchos casos, se ve apaciguada por la necesidad de "asegurarse un futuro", de encontrar un trabajo y de "ser alguien". La percepción del proceso de enseñanza-aprendizaje no es destacadamente positiva. Por fortuna, y pese a que algunos de ellos afirman "estar allí" por mera obligación, cierto es que muchos otros sí tienen un interés real en lograr unas metas, en su propio aprendizaje. No titubean, en ningún momento, a la hora de resaltar que el esfuerzo que los estudiantes invierten en aprender debe estar reconocido y valorado por los profesores, llegando incluso a etiquetar este hecho como un enclave imprescindible de la motivación ante el aprendizaje. Si bien la mayor parte de las atribuciones de su discurso se han focalizado en el papel del docente como factor que influencia notablemente la motivación ante el aprendizaje de estos estudiantes, en ningún caso debería obviarse que dicha labor ejercida por los profesionales está sujeta a la acción de numerosas variables que, habitualmente, escapan a su propio control. Entre ellas se encontrarían, a modo de ejemplo, las decisiones organizativas que se adoptan 
desde los centros, la estructura formativa actual para acceder al sistema universitario o los variados aspectos contextuales y organizativos que ejercen influencia sobre la labor de la enseñanza (Evers et al., 2016).

La motivación precede al aprendizaje y, a la luz de esta afirmación, no cabe duda alguna de que la motivación es un factor absolutamente crítico para la instrucción y el proceso de enseñanza-aprendizaje que se desarrolla en los centros de Educación Secundaria Obligatoria. Lo encontrado en este estudio resulta coincidente con los resultados de otros trabajos de investigación (García et al., 2015) desarrollados en otras etapas educativas diferentes tales como Educación Primaria e, incluso, a nivel universitario. Todo ello nos lleva a una profunda reflexión acerca del modelo educativo actual y los procedimientos de enseñanza que están implementándose en las aulas de Secundaria y Bachillerato. En el momento en que pasan a ser considerados sólo los resultados académicos, ejemplo de lo cual puede apreciarse en el estudio de Núñez et al. (2009), los jóvenes estudiantes tienden a manifestar conductas de aproximación y evitación (persiguen resultados favorables y evitan los negativos) que, en muchas ocasiones, pueden conducirles a elevados niveles de ansiedad, temor al fracaso y, en consecuencia, peores resultados académicos. Puede resultar conveniente, cuando los estudiantes no presentan una motivación intrínseca, proponerles el establecimiento de ciertos objetivos gracias a los que se vean en la tesitura de ponerse a prueba y superarse personal y académicamente (Karlen et al., 2019).

De igual modo que los jóvenes difieren en cuanto a competencias, conocimientos y habilidades, también son diferentes en el ámbito motivacional, una idea que requiere que el profesor parta de los motivos que cada alumno y alumna considera importantes en su proceso de aprendizaje. Resulta necesario, por ende, ampliar el cuerpo empírico para analizar, entre otros aspectos, la calidad de la instrucción y enseñanza que el alumnado recibe desde los centros de educación Secundaria y Bachillerato (Meece et al., 2006). De este modo, y de acuerdo con Veas, López-López et al. (2017), podría resultar fructífera la aplicación de ciertas reformas dentro de las aulas destinadas a promover la motivación del alumnado hacia el aprendizaje, el desarrollo de habilidades y la participación en la comunidad escolar. Aumentar el grado de motivación del alumnado requiere, en palabras de Urdan y Schoenfelder (2006), prestar especial atención a las características del ambiente en que estudian los estudiantes de Secundaria y Bachillerato ayudándoles a sentirse competentes, viendo el aprendizaje como un proceso continuo y satisfaciendo sus necesidades. Según Korpershoek et al. (2016), parece relevante identificar el tipo de perfil motivacional de cada alumno y alumna para adaptar las estrategias y procedimientos docentes a sus necesidades evitando, de esta manera, las altas tasas de fracaso hacia el aprendizaje y trabajando dentro de lo que podría denominarse como "zona de desarrollo próximo motivacional". Teniendo en cuenta que el alumnado está más motivado cuando las condiciones del ambiente de las clases son adecuadas, entonces el profesor debe asumir una parte de la responsabilidad personal de la motivación de estos (Murtagh, 2014) y, por ende, de la falta de esta que, hoy en día, reina en nuestros centros educativos.

Insistimos finalmente, en la línea del estudio de Barca et al. (2012), en la necesidad de indagar en los factores que inciden en un descenso de la motivación hacia el aprendizaje en las primeras etapas para trabajar de forma preventiva esta problemática que repercute en el rendimiento, tal y como han demostrado similares estudios (Akhtar et al., 2017) desarrollados en la misma etapa educativa que la del presente trabajo. Se intuye que muchos de ellos son los mismos, lo que debería llevar a una profunda reflexión sobre cómo estamos trabajando en nuestras escuelas, institutos y universidades.

Una de las implicaciones prácticas más relevantes a las que el estudio aquí planteado conduce, se refiere a las actuaciones que, desde los Departamentos de orientación y desde el ámbito familiar y escolar, pueden adoptarse para que el alumnado mantenga una motivación positiva ante sus aprendizajes. Desde estos campos de actuación, el cuidado de la autoestima, el desarrollo de competencias, el refuerzo de su sentido de la autosuficiencia y del esfuerzo personal 
conforman, todas ellas, dimensiones sobre las que resulta completamente necesario incidir. Téngase en cuenta que la motivación no depende de un único factor, sino que en ella se ven implicadas variables personales y contextuales de muy diversa índole. En consecuencia, las actuaciones y orientaciones que se les ofrezcan no deben desarrollarse desde un solo frente, sino en permanente colaboración entre los agentes que forman parte de los entornos habituales de estos estudiantes.

Este trabajo de investigación comporta, a su vez, una serie de limitaciones a destacar. En primer lugar, el estudio se encuentra condicionado a la homogeneidad en el criterio del curso para conformar los grupos de discusión. Además, y considerando la complejidad de las organizaciones escolares, la atribución al rol del docente de la responsabilidad prácticamente única de la motivación de su alumnado no refleja, en ningún caso, la realidad próxima. Aun con ello, la variedad de opiniones aquí expuestas sirve para, además de conocer la situación actual en esta materia de estudio, establecer un punto de partida para continuar indagando sobre los factores que influyen notablemente en la motivación del alumnado de estas etapas educativas. Convendría que futuros trabajos de investigación incorporasen el análisis de las percepciones de los docentes, a la par que, del alumnado, así como el estudio de otros elementos que pueden condicionar, directa o indirectamente, el desempeño profesional del profesorado.

\section{Referencias bibliográficas}

Akhtar, N.A., lqbal, M. y ljaz, A.T. (2017). Relationship between intrinsic motivation and students' academic achievement: A secondary level evidence. Bulletin of Education and Research, 39(2), 19-29. https://eric.ed.gov/?id=EJ1210159

Barca, A., Almeida, L. S., Porto, A.M., Peralbo, M. y Brenlla, J.C. (2012). Motivación escolar y rendimiento: impacto de metas académicas, de estrategias de aprendizaje y autoeficacia. Anales de Psicología, 28(3) 848-859. http://dx.doi.org/10.6018/analesps.28.3.156101

Bisquerra, R. (2004). Metodología de la investigación educativa. La Muralla.

Canales, M y Peinado, A. (1994). Grupos de discusión. En J.M. Delgado y J. Gutiérrez (Eds.), Métodos y técnicas cualitativas de investigación en ciencias sociales (pp.287-316). Síntesis Psicología.

Carey, M.N., Asbury, J.E. y Tolich, M. (2012). Focus group research. Left Coast Press.

Caso Niebla, J. y Hernández Guzmán, L. (2007). Variables que inciden en el rendimiento académico de adolescentes mexicanos. Revista Latinoamericana de Psicología, 39(3), 487-501. https://www.redalyc.org/articulo.oa?id=80539304

Cerrón Rojas, W. (2019). La investigación cualitativa en educación. Horizonte de la Ciencia, 9(17), 1-8. https://doi.org/10.26490/uncp.horizonteciencia.2019.17.510

Chien, C.F. y Hsieh, L.H.C. (2018). Exploring university students' achievement, motivation, and receptivity of Flipped Learning in an Engineering Mathematics course. International Journal of Online Pedagogy and Course Design, 8(4), 22-37. https://doi.org/10.4018//JOPCD.2018100102 
Cudney, E. y Ezzell J. (2018). Evaluating the impact of teaching methods on student motivation. Journal of STEM Education, 18(1), 32-49.

Río-Roberts, M. (2011). How I learned to conduct focus groups. Qualitative Report, 16(1), 312-315. https://doi.org/10.46743/2160-3715/2011.1057

Dogan, U. (2015). Student engagement, academic self-efficacy, and academic motivation as predictors of academic performance. Anthropologist, 20(3), 553-561. https://doi.org/10.1080/09720073.2015.11891759

Dotterer, A.M. y Lowe, K. (2011). Classroom context, school engagement, and academic achievement in early adolescence. Journal of Youth and Adolescence, 40(12), 1649-1660. https://doi.org/10.1007/s10964-011-9647-5

Džinović, V., Đević, R. y Đerić, I. (2019). The role of self-control, self-efficacy, metacognition, and motivation in predicting school achievement. Psihologija, 52(1), 35-52. https://doi.org/10.2298/PSI180202027D

Engels, M.C., Pakarinen, E., Lerkkanen, M.K. y Verschueren, K. (2019). Students' academic and emotional adjustment during the transition from primary to secondary school: a cross$\begin{array}{llll}\text { lagged study. Journal of School Psychology, 76, } & \text { 140-158. }\end{array}$ https://doi.org/10.1016/j.jsp.2019.07.012

Evers, A., Van der Heijden B. y Kreijns K. (2016). Organizational and task factors influencing teacher's, professional development at work. European Journal of Training and Development, 40, 36-55. https://eric.ed.gov/?id=EJ1086189

Froiland, J.M. y Oros, E. (2014). Intrinsic motivation, perceived competence and classroom engagement as longitudinal predictors of adolescent reading achievement. Educational Psychology, 34(2), 119-132. https://doi.org/10.1080/01443410.2013.822964

García, M.M., González, S.G. y Soto, J.G. (2015). Estudio exploratorio de intereses y motivación para la ejecución de tareas en alumnado de Educación Primaria de la provincia de Pontevedra. Revista de Investigación en Educación, 13(2), 256-270. http://education.esp.macam.ac.il/article/1311

Graham, S., Courtney, L., Tonkyn, A. y Marinis, T. (2016). Motivational trajectories for early language learning across the primary-secondary school transition. British Educational Research Journal, 42(4), 682-702. https://doi.org/10.1002/berj.3230

Grønkjær, M., Curtis, T., de Crespigny, C. y Delmar, C. (2011). Analysing group interaction in focus group research: Impact on content and the role of the moderator. Qualitative Studies, 2, 16-30. https://doi.org/10.7146/qs.v2i1.4273

Guay, F., Stupnisky, R., Boivin, M., Japel, C. y Dionne, G. (2019). Teachers' relatedness with students as a predictor of students' intrinsic motivation, self-concept, and Reading achievement. Early Childhood Research Quarterly, 48, 215-225. https://doi.org/10.1016/i.ecresq.2019.03.005

Guest, G., Namey, E. y McKenna, K. (2017). How many focus groups are enough? Building an evidence base for nonprobability sample sizes. Field Methods, 29, 3-22. https://doi.org/10.1177/1525822X16639015

Hanewald, R. (2013). Transition between primary and secondary school: Why it is important and how it can be supported. Australian Journal of Teacher Education, 38(1), 62-74. http://dx.doi.org/10.14221/ajte.2013v38n1.7 
Harreveld, B., Danaher, M., Lawson, C., Knight, B. A. y Busch, G. (2016). Constructing methodology for qualitative research. Palgrave Macmillan.

Huhtiniemi, M., Saakslahti, A., Watt, A. y Jaakkola, T. (2019). Associations among basic psychological needs, motivation, and enjoyment within Finnish physical education students. Journal of Sports Science \& Medicine, 18, 239-247.

Karlen, Y., Suterb, F., Hirta, C. y Merkib, K.M. (2019). The role of implicit theories in students' grit, achievement goals, intrinsic and extrinsic motivation, and achievement in the context of a long-term challenging task. Learning and Individual Differences, 74, 101757. https://doi.org/10.1016/j.lindif.2019.101757

Korpershoek, H., Harms, T., de Boer, H., van Kuijk, M. y Doolaard, S. (2016). A meta-analysis of the effects of classroom management strategies and classroom management programs on students' academic, behavioral, emotional, and motivational outcomes. Review of Educational Research, 86(3), 643-680. https://doi.org/10.3102/0034654315626799

Kunter, M., Klusmann, U., Baumert, J., Richter, D., Voss, T. y Hachfeld, A. (2013). Professional competence of teachers: effects on instructional quality and student development. Journal of Educational Psychology, 105(3), 805-820. https://doi.org/10.1037/a0032583

Leong, K.E., Tan, P.P., Lau, P.L. y Yong, S.L. (2018). Exploring the relationship between motivation and science achievement of Secondary students. Pertanika Journal of Social Sciences \& Humanities, 26(4), 2243-2258.

Meece, J., Anderman, E. y Anderman, L. (2006). Classroom goal structure, student motivation, and academic achievement. Annual review of psychology, 57, 505-528. https://doi.org/10.1146/annurev.psych.56.091103.070258

Meece, J., Herman, P. y McCombs, B. (2003). Relations of learner-centered teaching practices to adolescents' achievement goals. International Journal of Educational Research, 39(4-5), 457-475. https://doi.org/10.1016/j.ijer.2004.06.009

Mega, C., Ronconi, L. y De Beni, R. (2014). What makes a good student? How emotions, selfregulated learning, and motivation contribute to academic achievement. Journal of Educational Psychology, 106(1), 121-131. https://doi.org/10.1037/a0033546

Ministerio de Educación y Ciencia (2007). Datos básicos de la educación en España en el curso 2006/2007. Secretaría general técnica.

Monarca, H., Rappoport, S. y Fernández, A. (2012). Factores condicionantes de las trayectorias escolares en la transición entre enseñanza primaria y secundaria. Revista española de orientación $\quad y \quad$ psicopedagogía, https://doi.org/10.5944/reop.vol.23.num.3.2012.11461

Murtagh, L. (2014). The motivational paradox of feedback: teacher and student perceptions. The Curriculum Journal, 25(4), 516-541. https://doi.org/10.1080/09585176.2014.944197

Núñez, J.C., Cabanach, R.G., Rodríguez, S., González-Pienda, J.A. y Rosário, P. (2009). Perfiles motivacionales en estudiantes de secundaria: análisis de diferencias en estrategias cognitivas, estrategias de autorregulación y rendimiento académico. Revista Mexicana de Psicología, 26(1), 113-124. https://www.redalyc.org/articulo.oa?id=243016317011

Pouratashi, M. y Zhu, C. (2018). How students' views on educational factors influence their achievement motivation and learning approaches? Comparison of perspectives. International Journal of Africultural Management and Development, 8(4), 539-551. 
Reeve, J. y Lee, W. (2014). Students' classroom engagement produces longitudinal changes in classroom motivation. Journal of Educational Psychology, 106(2), 527-540. https://doi.org/10.1037/a0034934

Renaud, A., Guay, F., Talbot, D., Taylor, G. y Koestner, R. (2015). The relations between implicit intelligence beliefs, autonomous academic motivation, and school persistence intentions: A mediation model. Social Psychology of Education, 18(2), 255-272. https://doi.org/10.1007/s11218-014-9288-0

Roux, R. y Anzures, E.E. (2015). Estrategias de aprendizaje y su relación con el rendimiento académico en estudiantes de una escuela privada de educación media superior. Actualidades Investigativas en Educación, 15(1), 1-16. https://doi.org/10.15517/aie.v15i1.17731

Ruiz, L.S., Castro, Marianella y León, A.T. (2010). Transición a la secundaria: los temores y preocupaciones que experimentan los estudiantes de primaria. Revista Iberoamericana de Educación, 52(3), 1-13. https://doi.org/10.35362/rie5231793

Sánchez-Gómez, M.C., Hernández Ramos, J.P. y Costa, A. P. (2019). Investigación cualitativa en Ciencias Sociales: El caso de la educación. Fronteiras: Journal of Social, Technological and Environmental Science, 8(1), 12-17. http://dx.doi.org/10.21664/2238-8869.2019v8i1

Smit, K., de Brabander, C.J., Boekaerts, M. y Martens, R.L. (2017). The self-regulation of motivation: Motivational strategies as mediator between motivational beliefs and engagement for learning. International Journal of Educational Research, 82, 124-134. https://doi.org/10.1016/j.ijer.2017.01.006

Tasgin, A. y Coskun, G. (2018). The relationship between academic motivations and university students' attitudes towards learning. International Journal of Instruction, 11(4), 935-950.

Turner, J.C., Midgley, C., Meyer, D., Gheen, M., Anderman, E.M., Kang, Y. y Patrick, H. (2002). The classroom environment and students' reports of avoidance strategies in mathematics: a multimethod study. Journal of Educational Psychology, 94(1), 88-106. https://doi.org/10.1037/0022-0663.94.1.88

Urdan, T. y Schoenfelder, E. (2006). Classroom effects on student motivation: goal structures, social relationships, and competence beliefs. Journal of School Psychology, 44(5), 331349. https://doi.org/10.1016/i.jsp.2006.04.003

Vaugh, P. y Turner, C. (2016). Decoding via coding: Analyzing qualitative text data through thematic coding and survey methodologies. Journal of Library Administration, 56, 41-51. https://doi.org/10.1080/01930826.2015.1105035

Veas, A., López-López, J.A., Gilar, R., Miñano, P. y Castejón, J.L. (2017). Differences in cognitive, motivational and contextual variables between under-achieving, normally achieving, and over-achieving students: A mixed-effects analysis. Psicothema, 29(4), 533-538. https://doi.org/10.7334/psicothema2016.283

Fecha de entrada: 30 marzo 2020

Fecha de revisión: 25 mayo 2020

Fecha de aceptación: 08 junio 2020 